\title{
Photoautotrophic-heterotrophic biofilm communities: a laboratory incubator designed for growing axenic diatoms and bacteria in defined mixed-species biofilms
}

\author{
Matthias Buhmann, Peter G. Kroth and \\ David Schleheck* \\ Department of Biological Sciences, University of \\ Konstanz, D-78457 Konstanz, Germany.
}

\section{Summary}

Biofilm communities in the euphotic zone of aquatic habitats comprise photoautotrophic microorganisms, such as diatoms, green algae and cyanobacteria, which produce the organic carbon that fuels the life of a heterotrophic contingent of microorganisms, mostly bacteria. Such photoautotrophicheterotrophic mixed-species biofilms have received little attention in biofilm research due to a lack of suitable pure-culture laboratory model systems. However, they offer important insight into microbial population dynamics and community interactions during a biofilm-developmental process that shapes highly structured, extremely well-adapted microbial landscapes. Here, we report on the development of a sterile incubation chamber for growing and monitoring axenic phototrophic biofilms, i.e. a sterilizable, illuminated, continuous-flow system for a routine work with pure cultures. The system has been designed to simulate the growth conditions in the shallow, littoral zone of aquatic habitats (horizontal surface, submerged in water, illuminated, aerated). Additional features of the concept include automated photometrical monitoring of biofilm density (as biofilm turbidity), analysis via confocal microscopy, direct harvesting of cells, and options to control illumination, flow velocity, and composition of culture fluid. The application of the system was demonstrated in growth experiments using axenic diatom biofilms, or axenic diatom biofilms co-cultivated with different bacterial strains isolated from epilithic biofilms of an oligotrophic freshwater lake.

*For correspondence. E-mail david.schleheck@uni-konstanz.de; Tel. (+49) 07531 883270; Fax (+49) 07531 884047. Dr. David Schleheck, University of Konstanz, Department of Biological Sciences, P.O. box 649, Universitätsstrasse 10, Postfach 649, Konstanz, Germany, 78457.
Introduction

Every solid surface submerged in water and exposed to daylight in the natural aquatic environments is prone to colonization by phototrophic biofilms, complex communities of photoautotrophic and heterotrophic microorganisms conjointly embedded in a matrix of extracellular polymeric substances (e.g. Molino and Wetherbee, 2008; Roeselers et al., 2008). The extracellular polymeric substances mediate a firm attachment of the organisms onto the solid surface, and have many additional functions, such as protection from grazing, formation of a diffusion barrier and reservoir for recycling of nutrients (e.g. Wimpenny et al., 2000; Flemming et al., 2007). The photoautotrophic contingent of biofilm organisms fuels the whole biofilm community, while regeneration of nutrients is accomplished by a heterotrophic contingent of organisms (Cole, 1982; Canfield and Des Marais, 1993), e.g. in marine environments represented mainly by diatoms accompanied by heterotrophic bacteria (Marshall et al., 1971). Within such biofilms, the different organisms settle and live in distinct ecological niches, confined to their trophic level, gradients of light, nutrients and other growth factors present in the biofilm, and to their genetic repertoire for adaptation. This in combination with community interactions, cell death, dispersal or grazing, defines a biofilm-developmental process that finally shapes a matured, highly structured, extremely well-adapted 'microbial landscape' in each particular environmental setting (Battin et al., 2007).

Such phototrophic biofilms in shallow water habitats of all climate zones contribute significantly to ecosystem primary production and represent the basis of benthic food webs (e.g. Cahoon, 1999; Glud et al., 2002). Further, such biofilms can stabilize sediment surfaces and prevent their erosion (e.g. Lubarsky et al., 2010), and can determine the settlement of higher organisms such as macroalgal spores and invertebrate larvae (e.g. Qian et al., 2007). While being of great importance for aquatic ecosystems, phototrophic biofilms on man-made surfaces (biofouling) can cause severe economical damage, e.g. by increasing hydrodynamic drag on ship hulls or by covering equipment in aquaculture (e.g. Braithwaite and McEvoy, 2005; 
Schultz, 2007). To avoid biofouling, the submerged surfaces are often coated with paints that leach biocides onto the surface and into the open water, which can cause detrimental effects to the environment. Therefore, alternative strategies are needed (e.g. Brady, 2000; Chambers et al., 2006). The positive control of phototrophic biofilms can also be important for a range of biotechnological applications, e.g. for biofuel production (Roeselers et al., 2008; Tamburica et al., 2011).

The photoautotrophic organisms such as diatoms and the heterotrophic bacteria in their close association may influence each other (Cole, 1982; Murray et al., 1986; Fukami et al., 1997; Bruckner et al., 2008; Desbois et al., 2009), and these interactions can be exploited for both, inhibition of biofilm formation as well as establishment of stable biofilm communities. Therefore, more detailed studies of intraspecies and interspecies interactions during settlement and growth of phototrophic biofilms, and during their differentiation into matured biofilm communities, have a high potential to reveal novel targets for environmentally friendly antifouling strategies, and will hold important implications for the control of biofilms in biotechnological applications.

Methods to cultivate and study benthic phototrophic biofilms under controlled conditions are limited. To establish the biofilm-mode of growth in a laboratory, the biofilms are usually incubated under a continuous flow of culture medium (chemostatic culture) rather than in stationary culture (batch culture). The first condition represents the more common situation found in the aquatic environments, that is, convection of the open water that replenishes nutrients and removes metabolic products and dispersed cells. The established continuous-flow systems to study biofilms of heterotrophic bacteria, such as microscope-flow cells (e.g. Sternberg and Tolker-Nielsen, 2006) or more complex systems, such as the drip-flow biofilm reactor (Goeres et al., 2009), cannot be applied to phototrophic biofilms directly because of their limitations in light and/or gas exchange for photoautotrophic growth. A continuous-flow system that allows for the study of environmental phototrophic biofilms, but not of axenic phototrophic biofilms, has been developed by Zippel and colleagues (Zippel et al., 2007), in form of submerged glass slides fitted into horizontal flow lanes that are open to the top for aeration and illumination. This set-up can be used for microscopical analysis of undisturbed biofilms and included a device for monitoring the overall biofilm density, when measured photometrically as average light attenuation through the biofilm (biofilm turbidity).

In this work, we aimed to establish a continuous-flow system for phototrophic biofilms that simulates the growth conditions in the shallow, littoral zone of aquatic habitats and allows for the incubation of single-species and defined mixed-species biofilms, hence, a system that runs also under sterile conditions for a routine work with pure cultures. Therefore, we tried to meet the following criteria: (i) sterile incubation, (ii) horizontal surface substratum for biofilm formation, (iii) incubation under a continuous flow of a culture medium and air, (iv) defined illumination from the top for photosynthesis, and (v) possibilities for microscopical analysis of undisturbed biofilm and for sampling of biofilm biomass. Furthermore, a photometrical measurement of biofilm turbidity (Zippel et al., 2007) has also been included in our concept. The application of our novel incubation system was demonstrated in growth experiments using either the axenic freshwater diatom Planothidium sp., or Planothidium sp. co-inoculated with heterotrophic bacteria, all isolated from a freshwater epilithic biofilm of the littoral zone of Lake Constance. More detailed information on the Experimental procedures used with this incubation system may be found in Supporting information of the online version of this article.

\section{Results and discussion}

Design of the incubation system and its mode of operation

Key part of the novel incubation system was a horizontal flow lane (Fig. 1A) as the primary containment for biofilm incubation. Each flow lane was made of polycarbonate ( $76 \times 32 \mathrm{~mm}$ as base, $20 \mathrm{~mm}$ in height) into which a canal was shaped (40 mm in length, $16 \mathrm{~mm}$ in width). The canal was sealed at the base with a microscope-cover slide as the surface substratum for biofilm formation (total area, $580 \mathrm{~mm}^{2}$ ) and left open at the top (Fig. 1A). Hence, the flow lanes can be sterilized by autoclaving and fit well under microscopes.

For incubation, each flow lane was inserted into a secondary containment to provide the biofilms with illumination from the top, horizontal continuous flow of culture fluid, and sterile aeration (Fig. 1B). For illumination, three 'warm-white' diodes (LEDs) (colour temperature, $2900 \mathrm{~K}$; see also Supporting information) were fitted into the lid of each secondary containment (see Fig. 1B). We adjusted the light intensity to $55 \mu \mathrm{mol}$ photons $\mathrm{m}^{-2} \mathrm{~s}^{-1}$ at the surface for biofilm formation, as determined with a radiometer (see Experimental procedures). Furthermore, three visible-light sensors (peak sensitivity at $600 \mathrm{~nm}$; see Supporting information) were fitted into the base of each secondary containment for a photometrical monitoring of the overall biofilm turbidity (Zippel et al., 2007) in the particular sections of the flow lane (see Fig. 1B); the light sensors were connected to a datalogger (see Experimental procedures in Supporting information).

A continuous flow of sterile culture medium through each flow lane was achieved using multi-channel peristaltic pumps. With one channel, the feed medium was 

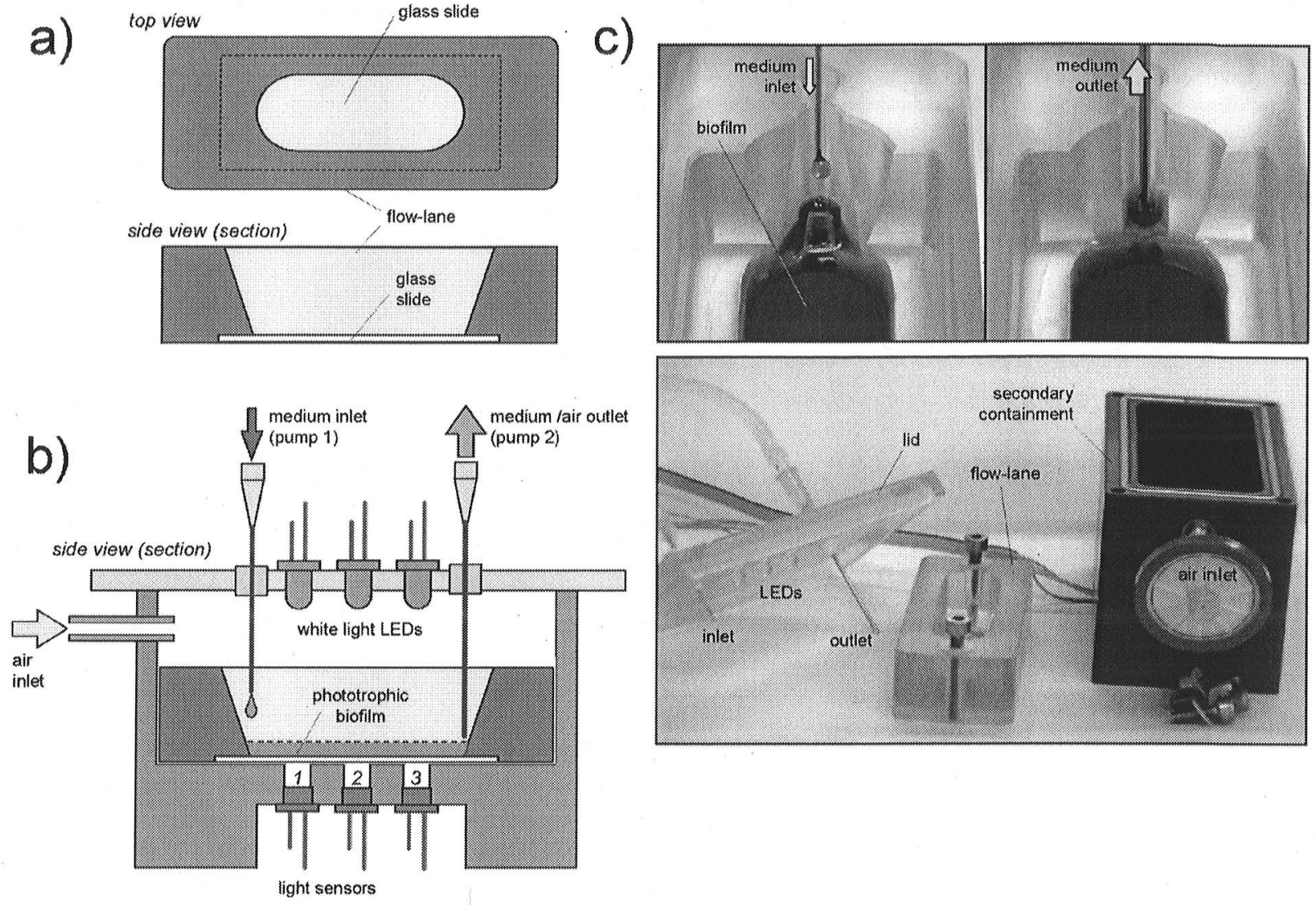

Fig. 1. Illustration of the novel incubation concept for phototrophic biofilms. The biofilms were grown on glass slides mounted into flow lanes (A) as the primary containments for biofilm incubation. Each flow lane was inserted into a secondary containment (B) that provided the biofilm with illumination for photosynthesis (using three white-light LEDs) and continuous flow of culture fluid and air (using peristaltic pumps). The optical density of the biofilm on the glass slide can be monitored photometrically (as biofilm turbidity), by using three light sensors fitted into the base of each secondary containment (B). The dimensions of the flow lanes (see text) were compatible with inverse-microscopy and normal microscopy (water immersion). See the text for further details on features of the set-up and operation of the system. The photographic illustrations (C) provide detailed views on the design of the medium inlet and outlet of the flow lanes (top), and on a disassembled set-up (bottom) comprising a flow lane, a secondary containment, and a lid with LEDs and syringe needles for medium inlet and outlet (see also text).

dripped into each flow lane at one end, through a syringe needle that was positioned well above the biofilm/glass surface (Fig. 1, inflow); this way entrance of microorganisms into the medium-feed line was prevented. With a second channel, the waste medium was continuously removed at the other end, through a siphon represented by a second syringe needle that was positioned right above the biofilm/glass-surface (Fig. 1B, outflow). With the flow rates adjusted in a way that the waste medium was removed much faster than the feed medium was provided, the set-up affected, firstly, that the culture fluid was kept at around the same level in the flow lanes (here, fluctuating at 3-4 mm in height) as excess fluid was removed via the siphon at intervals (overflow). Secondly, between these overflow intervals (i.e. during filling intervals), air was removed by the waste pump from the sealed, gas-tight secondary containment, leading to an inflow of fresh air through the sterile filter attached to the side of each secondary containment (Fig. 1B). Hence, the incubation concept provided a continuous exchange of both, liquid and gas phase, under sterile conditions.

\section{Validation of the incubation system}

Our initial tests of the incubation system (data not shown) revealed that axenically grown diatom biofilms were highly susceptible to the shear stress conditioned by the inflow (dripping) of culture fluid into the flow lanes: the drops detached diatoms and thereby carved grooves into the biofilms (e.g. see axenic biofilm in Fig. 4A, inset). Therefore, we modified the flow lanes. To absorb the liquid-shear at the inflow, the medium-inlet needle was pointed onto a 'drop break' that was added to each flow lane (see Fig. 1C, inlet). Furthermore, a 'spill-over' at the 
other end of the flow lane, into which the medium-outlet needle was positioned, was added (see Fig. 1C, outlet), in order to diminish the fluctuation of the water level during filling/overflow cycles (see above). The modifications ensured a uniform transfer of culture fluid through the flow lane, as was demonstrated when a pulse of crystal-violet staining solution was pumped through the flow lanes (Fig. 2A). In respect to liquid-shear force, this flow condition $\left(1.5 \mathrm{ml} \mathrm{h}^{-1}\right)$ and the improved design (Fig. 1C) allowed for a uniform formation of also axenically growing diatom biofilms (see below).

The stability of the sterilized set-up against microbial contamination was demonstrated in incubations with sterile nutrient broth [Luria-Bertani (LB) medium] (Fig. 2B). Sterilization of the growth systems was achieved as described in the Experimental procedures (see Supporting Information). No growth of microorganisms in the flow lanes was detected during a 10 day incubation, neither as biofilm turbidity when followed photometrically as shown here (Fig. 2B), nor by microscopy (not shown); also in the effluent medium, no microbial contamination was detectable, e.g. when testing by fluorescence microscopy (using nucleic-acid stain SYBR Green) and plating on LB medium (not shown). For comparison, if a flow lane was deliberately inoculated with bacteria (at day 6 in Fig. 2B), rapid formation of biofilm was detectable photometrically (Fig. 2B) as well as microscopically (not shown).

The visible-light sensors used for biofilm-turbidity measurement had their peak response at $600 \mathrm{~nm}$ (see Supporting information), thus produced an estimate of $\mathrm{OD}_{600}$, which is commonly used as a proxy for the growth of heterotrophic bacteria (e.g. Fig. 2B and ref. Schleheck et al., 2009). Photosynthetic microorganisms are commonly quantified by their chlorophyll a (chl-a) content. For the growth of the axenic diatoms used in this study, a linear correlation of biofilm turbidity (expressed in arbitrary units) and chlorophyll a content

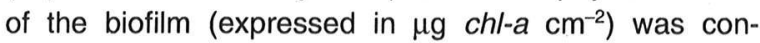
firmed (see Fig. S1 in Supporting information). Hence, the turbidity measurement generated a valid proxy for the formation of both, heterotrophic and phototrophic biomass in the incubation systems. Notably, the used visible-light sensors allowed for no distinction to be made between the growth of heterotrophs and phototrophs in mixed-species biofilms (see confocal microscopy, below).

\section{Experiments to demonstrate applications of the incubation system}

Growth dynamics of phototrophic biofilms in dependence on growth conditions were compared. In the example shown (Fig. 3), axenic Planothidium sp. biofilm was
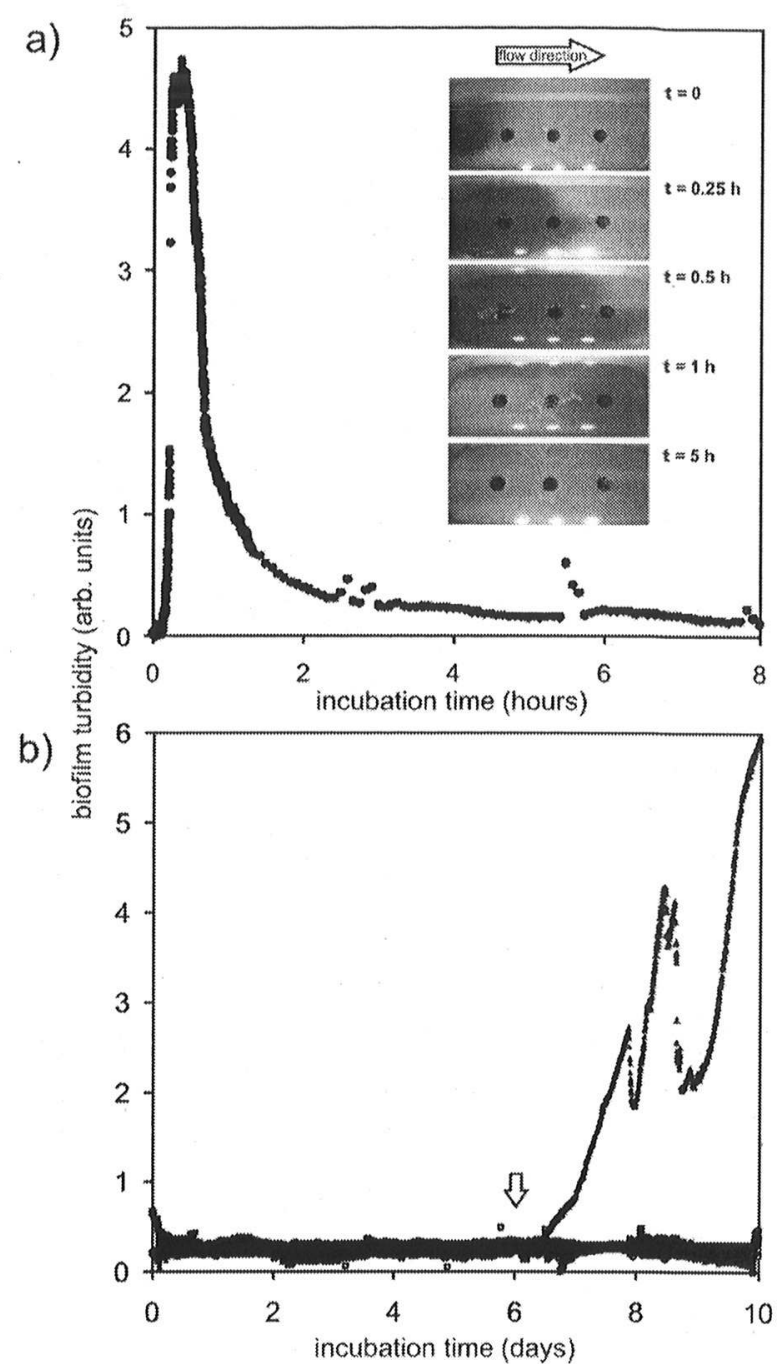

Fig. 2. Representative experiments to demonstrate the uniform transfer of culture fluid through a flow lane (A) and the stability of the incubation systems against microbial contamination (B).

A. A pulse of crystal-violet staining solution $(0.1 \mathrm{ml})$ was pumped with the feed-medium into a running system (standard flow rate,

$1.5 \mathrm{ml} \mathrm{h}^{-1}$ ) and the photometer readings were recorded (light sensor 1; see Fig. 1B); the inset photographs show the transition of a crystal-violet pulse through a flow lane at intervals.

B. Sterilized growth systems $(n=4)$ were run with sterile nutrient broth (LB medium; $1.5 \mathrm{ml} \mathrm{h}^{-1}$ ) and could be incubated for weeks (here, 10 days) without any sign of microbial contamination, as demonstrated here $(\mathrm{B})$ by photometrically monitoring the absence of biofilm turbidity ('baseline'). For comparison, a deliberately contaminated growth chamber (here, bacteria were injected into the feed line at day 6; arrow) exhibited a steep increase of biofilm turbidity, indicative of biofilm growth.

grown either under continuous flow, or without any flow of culture fluid (see Experimental procedures in Supporting information). As expected, the continuous medium supply produced a higher growth yield when observed by biofilm-turbidity measurement (Fig. 3), as well as a 


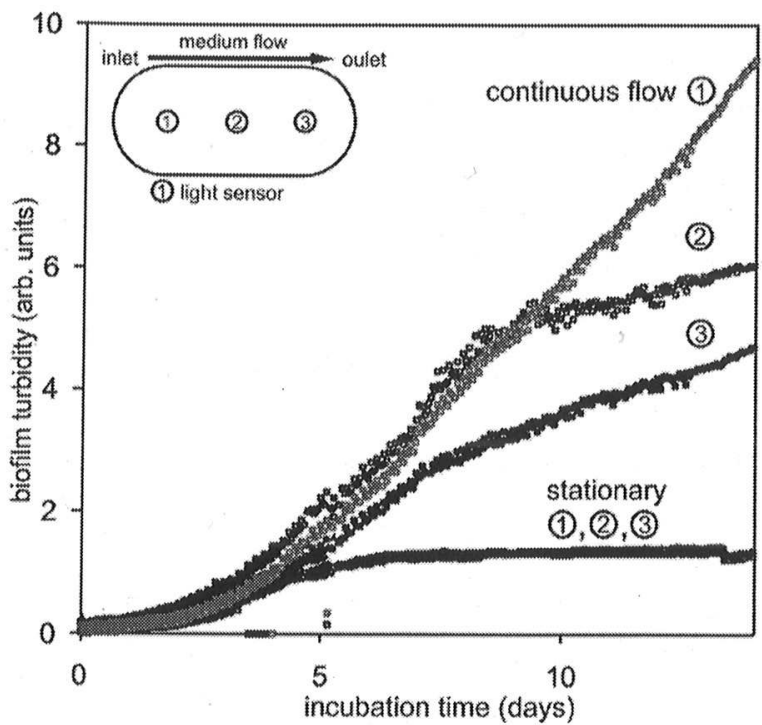

Fig. 3. Growth curves of axenic Planothidium sp. biofilms in flow lanes incubated under stationary condition (no liquid flow) and continuous flow of growth medium, as followed by biofilm-turbidity measurement. The inset illustrates the position of the three light sensors used for biofilm-turbidity measurements in each flow lane relative to the direction of inflow of fresh culture medium.

thicker biofilm when observed by microscopy (not shown). Without liquid flow, the biofilm growth curve (Fig. 3) reached a plateau after 5 days, i.e. the stationary phase, whereas growth under continuous flow proceeded until to the end of the experiment (15 days). However, the biofilm growth curves taken at the three different sections along the liquid flow (Fig. 3, inset) indicated that biofilm growth was also limited under continuous flow, progressively depending on the distance of the biofilm to the medium inlet (Fig. 3). This was attributed to a gradual depletion of nutrient(s) in the liquid stream along the flow lane. As calculated from the growth curve recorded at the inflow (light sensor No. 1, see Fig. 3), the maximum growth rate $(\mu)$ for the initial, exponential growth phase until day 5 was $0.014 \mathrm{~h}^{-1}\left(t_{\mathrm{D}}=2.1\right.$ days). For the following, linear growth phase, the linear growth rate $(\mathrm{m})$ was 0.7 turbidity-units per day. A linear growth of the biomass in phototrophic biofilms is a common observation (e.g. Kim et al., 2002), resulting from shading effects and light limitation of cells buried deeply within the biofilm in the later growth phase. The linear growth rate determined ( 0.7 turbidity-units day $\left.{ }^{-1}\right)$ could be translated into a rate of chlorophyll $a$ increase of approxi-

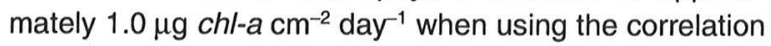
of biofilm turbidity and chl-a content of axenic Planothidium sp. biofilm (cf. Fig. S1). For comparison, when using published data on the chl-a increase during phototrophic biofilm formation in the natural environment under comparable conditions, e.g. on tiles in river-side flow channels (Battin et al., 2003; Roll et al., 2005; Besemer et al., 2007; Vinten et al., 2011), rates of chl-a increase in the range $0.03-2.0 \mu \mathrm{g} \mathrm{chl}-a \mathrm{~cm}^{-2}$ day $^{-1}$ could be calculated, dependent on, e.g. the nutrient supply or flow conditions used. Thus, the growth rate for the axenic phototrophic diatom biofilm in our incubation system was comparatively high, which likely reflected the continuous illumination of our biofilms in contrast to the day/night light regime for environmental biofilms, and the use of a synthetic freshwater-salts medium specifically for the growth of diatoms (DM medium; Beakes et al., 1988).

Another application of the incubation system was demonstrated when pre-grown diatom biofilms were treated with antifouling agents. In the example shown in the Supplemental information of this article (Fig. S2), a growing biofilm of Planothidium sp. was treated with a commercial preparation of a preservative for laboratory water baths, which stopped the growth and eradicated the biofilm (Fig. S2).

Finally, the utility of the incubation system for a work with defined mixed-species biofilms and for biofilm microscopy was demonstrated. In earlier work, it had been shown that diatom growth and/or aggregation can be enhanced by the presence of bacteria (e.g. Baker and Herson, 1978; Gawne et al., 1998; Grossart, 1999; Wigglesworth-Cooksey and Cooksey, 2005; Grossart et al., 2006; Grossart and Simon, 2007; Bruckner et al., 2008). Therefore, the effect of a co-inoculation of individual bacterial strains to axenically pre-grown diatom biofilms was examined. The diatom and bacterial strains tested (Fig. 4) were isolated from biofilms of the littoral zone of Lake Constance (see Experimental procedures in Supporting information). A general growth-enhancing effect of the presence of bacteria in Planothidium sp. biofilms was not observed; however, one bacterial isolate consistently enhanced Planothidium sp. biofilm formation, as determined by the dynamics of biofilm-turbidity increase after co-inoculation (Fig. 4A) as well as observed macroscopically (Fig. 4A, inset). In the example shown (Fig. 4A), the presence of Pseudomonas sp. strain 8c increased the biofilm turbidity formation threefold, i.e. the apparent linear growth rate of biofilm of Planothidium sp. with Pseudomonas sp. strain $8 \mathrm{c}$ was 2.3 turbidity-units day $^{-1}$ compared with a growth rate of about 0.7 turbidityunits day ${ }^{-1}$ for axenically-growing Planothidium sp. biofilm (Fig. 4) as observed typically under our cultivation conditions (cf. Figs 3 and S1). Microscopy of the biofilm surface (Fig. 4B) indicated that the co-cultivation of Planothidium sp. with Pseudomonas sp. strain $8 \mathrm{c}$ generated a more complex biofilm structure, i.e. biofilms in a 'wave-like' pattern in comparison with the unstructured layer of biofilm that was formed by Planothidium sp. alone. Differential microscopy of diatoms (red) and stained bacteria (green) at the base of the biofilm, i.e. near the glass- 

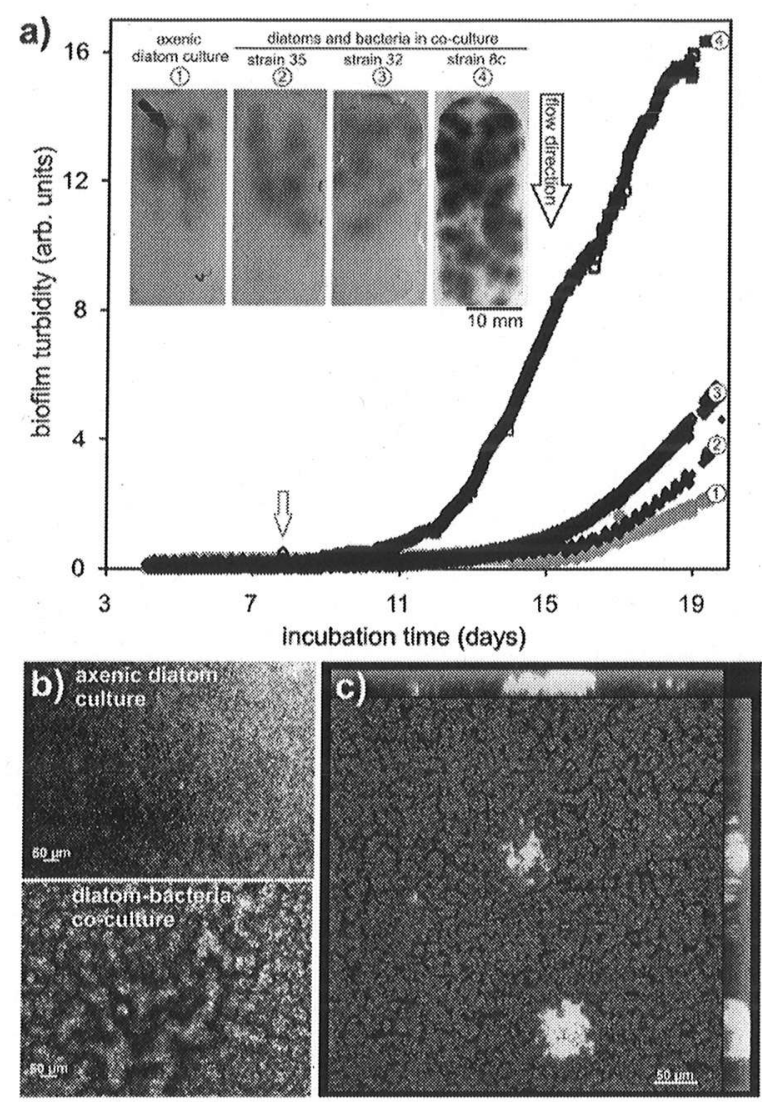

Fig. 4. Planothidium sp biofilm formation in dependence on co-cultivation of individual bacterial strains, as followed by turbidity measurement $(A)$ and macroscopical appearance of biofilms ( $A$, inset), and microscopical appearance $(B, C)$ of the diatom biofilm co-cultivated with Pseudomonas sp strain $8 \mathrm{c}$, as observed by brightfield microscopy (B) and confocal laser scanning microscopy (C). A. Axenic Planothidium sp. was inoculated into parallel systems $(n=4)$ and pre-grown for 8 days, after which different bacterial strains were co-inoculated (indicated by the arrow; see Experimental procedures); one biofilm was left untreated as axenic control. Indicated by an arrow in the photographic illustration ( $A$, inset) is the area where the axenic diatom biofilm had been stripped off the glass surface when the liquid-shear of inflowing medium was too high (see also text), while biofilms co-cultivated with bacteria remained attached.

B. Bright-field microscopical images of axenic Planothidium biofilm (top) in comparison with Planothidium biofilm co-cultivated with Pseudomonas sp strain $8 \mathrm{c}$ (bottom); the biofilms were observed at their surface by water-immersion microscopy.

C. Representative confocal microscopical image of a mixed-species biofilm of Planothidium sp. co-cultivated with Pseudomonas sp strain $8 \mathrm{c}$ at the base of the biofilm (near the glass surface), when diatoms were visualized by their chlorophyll auto-fluorescence (red) and bacteria by fluorescence staining (DiOC6 stain, green); note that the used inverse-confocal microscope was not able to resolve the biofilm composition in the deeper layers of biofilm (>50 $\mu \mathrm{m}$ depth), i.e. not at the biofilm surface.

surface using a confocal inverse-microscope, appeared that the bacterial contingent was represented in form of individual, discrete micro-colonies in the diatom biofilm (Fig. 4C).

\section{Conclusions}

The novel incubation concept for a routine work with pureculture phototrophic biofilms, as introduced here (Fig. 1), has been tested thoroughly (Figs $2-4$ and Supporting information), and may play a key role in the future characterization of surface colonization and growth dynamics of diatom biofilms. It will help to evaluate treatments for the control of diatom biofilm, and to identify, characterize and experimentally access interactions in diatom-bacteria mixed-species biofilms. The incubation concept at its present state of development, or with modifications, might also turn out to be useful for a work with other phototrophic biofilms, e.g. formed by green algae or cyanobacteria. Furthermore, the application of the novel incubation concept to reproducibly generate samples of phototrophic biofilms with known composition and highly defined cultivation history, might pave the way for a meaningful application of contemporary 'omics'-technologies, such as differential transcriptomics and proteomics, on phototrophic mixed-species biofilms.

\section{Acknowledgements}

We thank S. Auer, M. Weiland, M. Zimber and T. Trenker of the mechanical engineering and electronics workshop of the University of Konstanz for their excellent support. M.B. wishes to thank C. Rio-Bartulos for assistance in confocal microscopy, A. Ramsperger and K. Ojamäe for help with growth experiments, and $\mathrm{M}$. Windler for helpful discussions. This work was supported by funds of the University of Konstanz and the Konstanz Research School Chemical Biology (KoRSCB), the Deutsche Forschungsgemeinschaft (DFG grants SFB454 Bodensee-Littoral TP-B11 to P.G.K. and SCHL 1936/1-1 to D.S.), and by a 'Stiftung Umwelt and Wohnen' Grant of the University of Konstanz.

\section{References}

Baker, K.H., and Herson, D.S. (1978) Interactions between the diatom Thalassiosira pseudonana and an associated Pseudomonad in a marine culture system. Appl Environ Microbiol 35: 791-796.

Battin, T.J., Kaplan, L.A., Newbold, J.D., Cheng, X., and Hansen, C. (2003) Effects of current velocity on the nascent architecture of stream microbial biofilms. Appl Environ Microbiol 69: 5443-5452.

Battin, T.J., Sloan, W.T., Kjelleberg, S., Daims, H., Head, I.M., Curtis, T.P., and Eberl, L. (2007) Microbial landscapes: new paths to biofilm research. Nat Rev Microbiol 5: 76-81.

Beakes, G.W., Canter, H.M., and Jaworski, G.H.M. (1988) Zoospore ultrastructure of Zygorhizidium affluens and $Z$. planktonicum, two chytrids parasitising the diatom Asterionella formosa. Can J Bot 66: 1054-1067.

Besemer, K., Singer, G., Limberger, R., Chlup, A.K., Hochedlinger, G., Hödl, I., et al. (2007) Biophysical controls on community succession in stream biofilms. Appl Environ Microbiol 73: 4966-4974. 
Brady, R.F. (2000) Clean hulls without poisons: devising and testing nontoxic marine coatings. $J$ Coatings Technol 72: 45-56.

Braithwaite, R.A., and McEvoy, L.A. (2005) Marine biofouling on fish farms and its remediation. Adv Mar Biol 47: 215252.

Bruckner, C.G., Bahulikar, R., Rahalkar, M., Schink, B., and Kroth, P.G. (2008) Bacteria associated with benthic diatoms from Lake Constance: phylogeny and influences on diatom growth and secretion of extracellular polymeric substances. Appl Environ Microbiol 74: 7740-7749.

Cahoon, L.B. (1999) The role of benthic microalgae in neritic ecosystems. Oceanogr Mar Biol Annu Rev 37: 47-86.

Canfield, D.E., and Des Marais, D.J. (1993) Biogeochemical cycles of carbon, sulfur, and free oxygen in a microbial mat. Geochim Cosmochim Acta 57: 3971-3984.

Chambers, L.D., Stokes, K.R., Walsh, F.C., and Wood, R.J.K. (2006) Modern approaches to marine antifouling coatings. Surf Coat Technol 201: 3642-3652.

Cole, J.J. (1982) Interactions between bacteria and algae in aquatic ecosystems. Annu Rev Ecol Syst 13: 291-314.

Desbois, A., Mearns-Spragg, A., and Smith, V. (2009) A fatty acid from the diatom Phaeodactylum tricornutum is antibacterial against diverse bacteria including multi-resistant Staphylococcus aureus (MRSA). Mar Biotechnol 11: 45-52.

Flemming, H.C., Neu, T.R., and Wozniak, D.J. (2007) The EPS matrix: the 'house of biofilm cells. $J$ Bacteriol 189: 7945-7947.

Fukami, K., Nishijima, T., and Ishida, Y. (1997) Stimulative and inhibitory effects of bacteria on the growth of microalgae. Hydrobiologia 358: 185-191.

Gawne, B., Wang, Y., Hoagland, K., and Gretz, M.R. (1998) Role of bacteria and bacterial exopolymer in the attachment of Achnanthes longipes. Biofouling 13: 137-156.

Glud, R., Kühl, F., Wenzhöfer, F., and Rysgaard, S. (2002) Benthic diatoms of a high Arctic fjord (Young Sound, NE Greenland): importance for ecosystem primary production. Mar Ecol Prog Ser 238: 15-29.

Goeres, D.M., Hamilton, M.A., Beck, N.A., BuckinghamMeyer, K., Hilyard, J.D., Loetterle, L.R., et al. (2009) A method for growing a biofilm under low shear at the airliquid interface using the drip flow biofilm reactor. Nat Protoc 4: 783-788.

Grossart, H.-P. (1999) Interactions between marine bacteria and axenic diatoms (Cylindrotheca fusiformis, Nitzschia laevis and Thalassiosira weissflogii) incubated under various conditions in the lab. Aquat Microb Ecol 19: 1-11.

Grossart, H.-P., and Simon, M. (2007) Interactions of planktonic algae and bacteria: effects on algal growth and organic matter dynamics. Aquat Microb Ecol 47: 163-176.

Grossart, H.-P., Czub, G., and Simon, M. (2006) Algaebacteria interactions and their effects on aggregation and organic matter flux in the sea. Environ Microbiol 8: 14622920.

Kim, N.-J., Suh, I.S., Hur, B.-K., and Lee, C.-G. (2002) Simple monodimensional model for linear growth rate of photosynthetic microorganisms in flat-plate photobioreactors. J Microbiol Biotechnol 12: 962-971.

Lubarsky, H.V., Hubas, C., Chocholek, M., Larson, F., Manz, W., Paterson, D.M., and Gerbersdorf, S.U. (2010) The stabilisation potential of individual and mixed assemblages of natural bacteria and microalgae. PLOS ONE 5: e13794.

Marshall, K., Stout, R., and Mitchell, R. (1971) Mechanims of the initial events in the sorption of marine bacteria to surfaces. Microbiology 68: 337-348.

Molino, P.J., and Wetherbee, R. (2008) The biology of biofouling diatoms and their role in the development of microbial slimes. Biofouling 24: 365-379.

Murray, R.E., Cooksey, K.E., and Priscu, J.C. (1986) Stimulation of bacterial DNA synthesis by algal exudates in attached algal-bacterial consortia. Appl Environ Microbiol 52: 1177-1182.

Qian, P.-Y., Lau, S.C.K., Dahms, H.-U., Dobretsov, S., and Harder, T. (2007) Marine biofilms as mediators of colonization by marine macroorganisms: implications for antifouling and aquaculture. Mar Biotechnol 10: 1-12.

Roeselers, G., Loosdrecht, M.C., and Muyzer, G. (2008) Phototrophic biofilms and their potential applications. J Appl Phycol 20: 227-235.

Roll, S.K., Diehl, S., and Cooper, S.D. (2005) Effects of grazer immigration and nutrient enrichment on an open algae-grazer system. Oikos 108: 386-400.

Schleheck, D., Barraud, N., Klebensberger, J., Webb, J.S., McDougald, D., Rice, S.A., and Kjelleberg, S. (2009) Pseudomonas aeruginosa PAO1 preferentially grows as aggregates in liquid batch cultures and disperses upon starvation. PLoS ONE 4: e5513.

Schultz, M.P. (2007) Effects of coating roughness and biofouling on ship resistance and powering. Biofouling 23: 331-341.

Sternberg, C., and Tolker-Nielsen, T. (2006) Growing and analyzing biofilms in flow cells. Curr Protoc Microbiol. Chapter 1: Unit 1B.2.

Tamburica, B., Zemichaela, F.W., Crudgea, P., Maitlanda, G.C., and Hellgardt, K. (2011) Design of a novel flat-plate photobioreactor system for green algal hydrogen production. Int J Hydrogen Energy 36: 6578-6591.

Vinten, A.J., Artz, R.R., Thomas, N., Potts, J.M., Avery, L., Langan, S.J., et al. (2011) Comparison of microbial community assays for the assessment of stream biofilm ecology. J Microbiol Methods 85: 190-198.

Wigglesworth-Cooksey, B., and Cooksey, K. (2005) Use of fluorophore-conjugated lectins to study cell-cell interactions in model marine biofilms. App/ Environ Microbiol 71: 428-435.

Wimpenny, J., Manz, W., and Szewzyk, U. (2000) Heterogeneity in biofilms. FEMS Microbiol Rev 24: 661-671.

Zippel, B., Rijstenbil, J., and Neu, T.R. (2007) A flow-lane incubator for studying freshwater and marine phototrophic biofilms. J Microbiol Methods 70: 336-345.

\section{Supporting information}

Additional Supporting Information may be found in the online version of this article:

Fig. S1. Reproducibility of diatom-biofilm growth as followed by turbidity measurement (A) and linear correlation of turbidity and chlorophyll a (chl-a) content of the biofilms (B). Growth systems were inoculated in parallel and incubated under continuous flow of culture fluid, in this example (A) axenic 
marine diatom $P$. tricornutum $(n=3)$. For the example shown here $(A)$, the turbidity readings were recorded every $10 \mathrm{~min}$, and such 'oversampling' of data appeared an oscillation of the baseline that represented the periodical exchange of culture fluid in the flow lanes (i.e. the filling/overflow intervals, see text); a short period of tenfold-increased medium flow (30 min, $15 \mathrm{ml} \mathrm{h}^{-1}$ ) is indicated by an arrow. (B) A sufficiently linear correlation $\left(R^{2}=0.97\right)$ between biofilm turbidity reading and chlorophyll a content throughout growth of the biofilms was confirmed when individual biofilms were sacrificed at intervals for chlorophyll a determination (see Experimental procedures). In the example shown (B), axenic freshwater diatom Planothidium sp. was used $(n=6)$. We calculated with a Planothidium-specific conversion factor of 1.5 for changing arbitrary biofilm turbidity-units (B) into chl-a content per surface area $\left(\mu \mathrm{g} \mathrm{chl}-\mathrm{a} \mathrm{cm}^{-2}\right)$ for the calculation of Planothidium $\mathrm{sp}$. biofilm growth rates in later experiments (see main text).

Fig. S2. Effect of treatment of pre-established Planothidium sp. biofilms with an algicidal agent, as followed by biofilmturbidity measurement (left) and macroscopical appearance of the biofilm (right). The photographic illustrations refer to the time points indicated in the biofilm-turbidity measurement. The treatment ( 3 days) resulted in bleaching of diatom biofilm from naturally brown to colourless during the following incubation (4 days) with growth medium (Fig. 5). The eradication of phototrophic biofilm was resembled in the biofilm-turbidity measurement (Fig. 5) as a peak observed directly after the start of the treatment ( 1 day), followed by stagnation (2 days) and gradual decrease (4 days) of the biofilm turbidity to approximately $75 \%$ of the maximal value before treatment. The remainder turbidity observed $\left(\approx \mathrm{OD}_{600}\right)$ was attributed to the opacity of debris of bleached diatom biofilm in the flow lane, as indicated by microscopy (not shown).

Fig. S3. Supplemental information on the characteristics of the LEDs used. Relevant excerpts are shown of the Technical Datasheet for LED 'LM560A' provided by the manufacturer Seoul Semiconductor, Korea.

Please note: Wiley-Blackwell are not responsible for the content or functionality of any supporting materials supplied by the authors. Any queries (other than missing material) should be directed to the corresponding author for the article. 